Article

\title{
Association of Genetic Variation in the Epithelial Sodium Channel Gene with Urinary Sodium Excretion and Blood Pressure
}

\author{
Yoon Jung Yang ${ }^{1,+(\mathbb{D}, \text { Jihye Kim }}{ }^{2,+}$ and Chang Keun Kwock ${ }^{3, *}$ \\ 1 Department of Foods and Nutrition, College of Natural Sciences, Dongduk Women's University, \\ Seoul 02748, Korea; yjyang@dongduk.ac.kr \\ 2 Department of Preventive Medicine, College of Medicine, Hanyang University, Seoul 04764, Korea; \\ eluzai81@hanyang.ac.kr \\ 3 Nutrition and Diet Research Group, Korea Food Research Institute, Jeollabuk-do 55365, Korea \\ * Correspondence: kwock@kfri.re.kr; Tel.: +82-063-219-9177 \\ + These authors contributed equally to this work.
}

Received: 26 March 2018; Accepted: 11 May 2018; Published: 14 May 2018

\begin{abstract}
This study was performed to investigate whether genetic variation in the epithelial sodium channel $(\mathrm{ENaC})$ is associated with 24-h urinary sodium excretion and blood pressure. A total of 3345 participants of the KoGES_Ansan and Ansung study were eligible for this study. Genomic DNA samples were isolated from peripheral blood and genotyped on the Affymetrix Genome-Wide Human SNP Array 5.0. Thirty-four single nucleotide polymorphisms (SNPs) were extracted for gene regions (SCNN1A, SCNN1B, and SCNN1G) as additive components by using Plink. Twenty-four-hour sodium excretions were estimated from spot urine samples using the Tanaka formula. The general linear model (GLM) was applied to assess the association between SNPs and urinary sodium excretion or blood pressure. In the SCNN1G gene, six SNPs (rs4073291, rs12934362, rs7404408, rs4494543, rs5735, and rs6497657) were significantly different in 24-h urinary sodium excretion according to gene variants. However, no difference was found in blood pressure among participants with gene variants of ENaC. Our finding indicated that 24-h urinary sodium excretions were different according to variants of the SCNN1G gene in large samples. Further studies to replicate these findings are warranted.
\end{abstract}

Keywords: ENaC; genetic variant; SCNN1A; SCNN1B; SCNN1G; blood pressure; 24-h urinary sodium excretion

\section{Introduction}

The epithelial sodium channel $(\mathrm{ENaC})$ is expressed in several excretory organs such as the salivary glands, kidney, and lung [1-6]. There are four ENaC channel subunits, i.e., $\alpha, \beta, \gamma$, and $\delta$, in humans [7], which are encoded by four genes (SCNN1A, SCNN1B, SCNN1G, and SCNN1D, respectively).

$\mathrm{ENaC}$ is located in the distal nephron and plays a crucial role in controlling sodium balance. ENaC is mostly expressed in the luminal membrane of connecting tubule cells and plays an important role in renal sodium reabsorption and excretion $[3,4]$. Excessive sodium reabsorption by the kidney has been known to increase the risk of hypertension. In the kidney, the final control of sodium reabsorption takes place in the distal nephron through ENaC [4]. Liddle's syndrome, a hereditary form of hypertension due to gain-of-function mutations in the genes coding for ENaC subunits, has shown the key role of $\mathrm{ENaC}$ in the sodium balance [8].

$\mathrm{ENaC}$ is located within taste cell membranes and is the primary mediator of salt taste. ENaC seems to be responsible for the appetitive behavioral responses caused by salt taste [1,2]. Taste perception plays 
an important role in determining individual food preferences, which may influence nutritional status and the risk of chronic disease [9]. If so, salt taste perception may affect sodium intake, which may further affect blood pressure.

$\mathrm{ENaC}$ has been reported to play an important role in lung fluid clearance [10]. SCNN1A appears to be essential in fluid transport in the lung. Genetic variants of SCNN1A were associated with the risk of neonatal respiratory distress syndrome [6].

Previous studies have suggested that genetic variants of $\mathrm{ENaC}$ subunits may influence blood pressure or hypertension [11]. The results are controversial. To our knowledge, the 24-h urinary sodium excretion by genetic variants of ENaC subunits has not been investigated yet among Asian populations. Therefore, this study was performed to determine whether genetic variation in ENaC (SCNN1A, $S C N N 1 B$, and SCNN1G) is associated with 24-h urinary sodium excretion and blood pressure in a Korean adult population.

\section{Materials and Methods}

\subsection{Study Population}

The KoGES_Ansan and Ansung study, an ongoing community-based cohort, was initiated to investigate the trends in diabetes and the related risk factors as part of the Korean Genome Epidemiology Study (KoGES) in 2001. At baseline, 10,038 participants aged 40-69 years were recruited from two communities, Ansan $(n=5020)$ and Ansung $(n=5018)$. Ansan is an urban area located in the southwest of Seoul (the capital of Korea), and Ansung is a rural area located south of Seoul. The baseline examination was conducted between 2001 and 2002, and the participants were followed up biennially. The details of the study design and procedures are described in a previous report [12].

DNA samples obtained from 10,004 participants were genotyped. After performing the sample and SNP quality control, 8840 participants were included in the data analysis. The detailed process has been described elsewhere [13]. Among 8840 participants, we excluded participants who had no information for spot urine samples $(n=4425)$. In addition, participants who were diagnosed by a doctor as having hypertension, diabetes, myocardial infarction, heart failure, coronary artery disease, stroke, renal disease, or cancer were excluded. Finally, a total of 3345 participants were included in the final data analysis. This study was approved by the electronic institutional review board (e-IRB) of the Korea National Institute for Bioethics Policy (KoNIBP).

\subsection{General Characteristics and Anthropometric Variables}

Interview-based questionnaires were performed by interviewers trained with a standardized manual to obtain demographic information, medical history, family history, and lifestyles. Weight was measured in kilograms to the nearest $0.1 \mathrm{~kg}$, and height was measured within $0.1 \mathrm{~cm}$ without shoes. Body mass index (BMI) was defined as the body weight in kilograms divided by the square of the height in meters $\left(\mathrm{kg} / \mathrm{m}^{2}\right)$. Systolic blood pressure (SBP) and diastolic blood pressure (DBP) were measured at least twice in a supine position, and then an average value was calculated.

\subsection{Collection of Urinary Samples and Estimation of 24-h Sodium Excretion}

Urine specimens were self-collected in urine cups, and administrators transferred the urine into conical tubes, which were then sent to a central laboratory (Seoul Clinical Laboratories, Seoul, Korea) for measuring the quantities of sodium. Estimation of 24-h sodium excretion was performed using the Tanaka formula [14].

\subsection{Genotyping}

Genomic DNA samples were isolated from peripheral blood and genotyped on the Affymetrix Genome-Wide Human SNP Array 5.0 (Affymetrix Inc., Santa Clara, CA, USA). Genotype calling was conducted with the Bayesian Robust Linear Modeling using the Mahalanobis Distance (BRLMM) 
Genotyping Algorithm (DNA Link, Seoul, Korea) for 500,568 SNPs, and 352,228 SNPs remained after quality control. SNP imputation was conducted using the IMPUTE program $[15,16]$. Based on NCBI build 36 and dbSNP build 126, the JPT and CHB in HAPMAP were used as a reference panel comprising 3.99 million SNPs (HapMap release 22). All genetic variants were examined for Hardy-Weinberg equilibrium. Markers with Hardy-Weinberg equilibrium $p$ value $<10^{-6}$ were discarded. More details on the genotype calling, quality-control, and imputation processes are described in a previous study [13].

\subsection{Statistical Analysis}

By using Plink (http:/ / pngu.mgh.harvard.edu/ purcell/plink2/), we extracted SNP genotype data for gene regions (SCNN1A, SCNN1B, and $S C N N 1 G)$ as additive $(0,1$, and 2$)$ components. A total of 34 SNPs were identified. Association analyses between SNPs and urinary sodium excretion or blood pressure were conducted using SAS software (version 9.3; SAS Institute, Cary, NC, USA).

The general linear model (GLM) was used to assess the association between SNPs and urinary sodium excretion or blood pressure according to the number of minor alleles after being adjusted for age, sex, BMI, and smoking status (non-smoker and current smoker). $p$-Values $<0.05$ were considered significant. Tukey's multiple comparison test was applied to search for specific differences between pairs of groups at $p<0.05$. The linear trend test was performed by GLM in an additive genetic model with 1 degree of freedom.

\section{Results}

Table 1 shows the general characteristics of the study subjects. The mean ages of male and female subjects were 51.9 years and 51.0 years, respectively. The means of BMI for male and female subjects were $24.0 \mathrm{~kg} / \mathrm{m}^{2}$ and $24.5 \mathrm{~kg} / \mathrm{m}^{2}$. The proportions of the subjects with a family history of hypertension were $14.3 \%$ for male subjects and $15.6 \%$ for female subjects. Table 2 demonstrates allele distributions of SNPs in putative salt taste receptors within the study population. Major allele homozygous, heterozygous, and minor allele homozygous are presented as MM, Mm, and mm, respectively.

Table 1. General characteristics of the study subjects.

\begin{tabular}{lcc}
\hline \multicolumn{1}{c}{ Characteristics } & Men & Women \\
\hline$n$ & 1446 & 1899 \\
\hline Age (year) & $51.9 \pm 8.8^{1}$ & $51.0 \pm 8.8$ \\
\hline Income, $\geq 2,000,000 \mathrm{KRW}(\%)$ & 33.3 & 30.0 \\
\hline Cigarette smoking, current (\%) & 55.7 & 3.6 \\
\hline Alcohol drinking, current (\%) & 68.9 & 25.3 \\
\hline Regular exercise, yes (\%) & 10.3 & 15.2 \\
\hline BMI (kg/m $\left.{ }^{2}\right)^{2}$ & $24.0 \pm 3$ & $19.5 \pm 3.2$ \\
\hline Energy intake (kcal/day) & $2115.5 \pm 756.1$ & $113.9 \pm 17.4$ \\
\hline Family history of hypertension (\%) & 14.3 & $72.0 \pm 11.2$ \\
\hline Blood pressure (mmHg) & & \\
$\quad$ Systolic blood pressure & $116.4 \pm 15.8$ & 844.3 \\
\hline Diastolic blood pressure & $75.4 \pm 10.4$ & 1980.2 \\
\hline
\end{tabular}

${ }^{1}$ Mean \pm SD; ${ }^{2}$ BMI: body mass index. 
Table 2. The allele distributions of SNPs in the epithelial sodium channel $(\mathrm{ENaC})$ within the study population.

\begin{tabular}{|c|c|c|c|c|c|c|}
\hline \multirow{2}{*}{ Gene } & \multirow{2}{*}{ SNP } & \multicolumn{3}{|c|}{ Genotype $^{1}$} & \multirow{2}{*}{ Alteration } & \multirow{2}{*}{ MAF (\%) } \\
\hline & & MM & $\mathrm{Mm}$ & $\mathrm{mm}$ & & \\
\hline \multirow{4}{*}{ SCNN1A } & rs13306613 & 3039 (90.85) & $296(8.85)$ & $10(0.30)$ & $\mathrm{C}>\mathrm{T}$ & 5 \\
\hline & rs7956915 & $1314(39.29)$ & $1564(46.77)$ & $466(13.94)$ & $\mathrm{G}>\mathrm{A}$ & 37 \\
\hline & rs4149621 & $2823(92.10)$ & $238(7.77)$ & $4(0.13)$ & $\mathrm{T}>\mathrm{C}$ & 4 \\
\hline & rs11064153 & $1391(41.58)$ & $1538(45.98)$ & $416(12.44)$ & $\mathrm{C}>\mathrm{T}$ & 36 \\
\hline \multirow{8}{*}{ SCNN1B } & rs7205273 & $1731(51.75)$ & $1334(39.88)$ & $280(8.37)$ & $\mathrm{C}>\mathrm{T}$ & 27 \\
\hline & rs7190829 & $2152(64.33)$ & 1043 (31.18) & $150(4.48)$ & $\mathrm{A}>\mathrm{G}$ & 19 \\
\hline & rs8055868 & $2156(64.49)$ & $1030(30.81)$ & $157(4.70)$ & $\mathrm{G}>\mathrm{A}$ & 19 \\
\hline & rs8044970 & $1064(31.85)$ & $1622(48.55)$ & $655(19.6)$ & $\mathrm{T}>\mathrm{G}$ & 44 \\
\hline & rs152733 & $1100(32.88)$ & $1642(49.09)$ & 603 (18.03) & $\mathrm{T}>\mathrm{C}$ & 42 \\
\hline & rs239350 & $2682(88.46)$ & $336(11.08)$ & $14(0.46)$ & $\mathrm{C}>\mathrm{T}$ & 6 \\
\hline & rs889299 & 1704 (50.97) & $1346(40.26)$ & $293(8.76)$ & $\mathrm{G}>\mathrm{A}$ & 30 \\
\hline & rs11074555 & $1921(57.43)$ & $1208(36.11)$ & $216(6.46)$ & $\mathrm{T}>\mathrm{C}$ & 25 \\
\hline \multirow{22}{*}{ SCNN1G } & rs4073291 & 2475 (73.99) & $803(24.01)$ & $67(2.00)$ & $\mathrm{A}>\mathrm{C}$ & 14 \\
\hline & rs12934362 & $2475(74.84)$ & 769 (23.25) & $63(1.91)$ & $\mathrm{T}>\mathrm{C}$ & 13 \\
\hline & rs7404408 & $2475(74.21)$ & 793 (23.78) & $67(2.01)$ & $\mathrm{C}>\mathrm{T}$ & 14 \\
\hline & rs4494543 & $2475(74.21)$ & 793 (23.78) & $67(2.01)$ & $A>G$ & 14 \\
\hline & rs5735 & $2462(75.61)$ & $734(22.54)$ & $60(1.84)$ & $\mathrm{T}>\mathrm{C}$ & 13 \\
\hline & rs6497657 & $2457(75.90)$ & $721(22.27)$ & $59(1.82)$ & $\mathrm{T}>\mathrm{C}$ & 13 \\
\hline & rs4299163 & 2796 (91.07) & $267(8.70)$ & $7(0.23)$ & $\mathrm{G}>\mathrm{C}$ & 5 \\
\hline & rs11643777 & $2962(91.76)$ & $261(8.09)$ & $5(0.15)$ & $C>G$ & 4 \\
\hline & rs4260062 & $2797(89.36)$ & 325 (10.38) & $8(0.26)$ & $\mathrm{T}>\mathrm{C}$ & 6 \\
\hline & rs5740 & $2798(89.08)$ & 334 (10.63) & $9(0.29)$ & $\mathrm{G}>\mathrm{A}$ & 6 \\
\hline & rs4499238 & $2767(84.36)$ & 491 (14.97) & $22(0.67)$ & $\mathrm{C}>\mathrm{T}$ & 9 \\
\hline & rs4470152 & $2798(87.63)$ & 384 (12.03) & $11(0.34)$ & $\mathrm{G}>\mathrm{T}$ & 7 \\
\hline & rs4309398 & $2798(87.79)$ & 379 (11.89) & $10(0.31)$ & $\mathrm{C}>\mathrm{T}$ & 7 \\
\hline & rs4341748 & $2798(87.82)$ & 378 (11.86) & $10(0.31)$ & $G>A$ & 7 \\
\hline & rs11648257 & $2963(89.22)$ & 349 (10.51) & $9(0.27)$ & $\mathrm{C}>\mathrm{T}$ & 6 \\
\hline & rs9941210 & 2963 (89.19) & $350(10.54)$ & $9(0.27)$ & $\mathrm{G}>\mathrm{T}$ & 6 \\
\hline & rs4499239 & $2963(89.17)$ & 351 (10.56) & $9(0.27)$ & $C>G$ & 6 \\
\hline & rs13306653 & $2963(88.90)$ & $361(10.83)$ & $9(0.27)$ & $\mathrm{G}>\mathrm{A}$ & 6 \\
\hline & rs11643517 & $2963(88.77)$ & $366(10.96)$ & $9(0.27)$ & $A>G$ & 6 \\
\hline & rs5723 & $2961(88.73)$ & 367 (11.00) & $9(0.27)$ & $C>G$ & 6 \\
\hline & rs3026 & $2962(88.74)$ & 367 (10.99) & $9(0.27)$ & $\mathrm{T}>\mathrm{G}$ & 6 \\
\hline & rs9930846 & $2962(88.74)$ & 367 (10.99) & $9(0.27)$ & $\mathrm{T}>\mathrm{C}$ & 6 \\
\hline
\end{tabular}

1 All values are presented as $n$ (\%); MM: major allele homozygous; Mm: heterozygous; mm: minor allele homozygous; MAF: minor allele frequency.

The associations of genetic variation in the $\mathrm{ENaC}$ gene with estimated 24-h urinary sodium excretion obtained with the Tanaka formula are presented in Table 3. In the SCNN1G gene, individuals homozygous for the $C$ allele of rs4073291 (A>C) showed lower 24-h urinary sodium excretion than those with either the AA or AC genotype. Individuals with homozygous for the $C$ allele of rs 12934362 (T>C) showed lower 24-h urinary sodium excretion than those with either the TT or TC genotype. Individuals homozygous for the $\mathrm{T}$ allele of rs7404408 (C>T) showed lower 24-h urinary sodium excretion than those with either the CC or CT genotype. Individuals homozygous for the $\mathrm{G}$ allele of rs4494543 (A>G) showed lower 24-h urinary sodium excretion than those with either the AA or AG genotype. Individuals homozygous for the C allele of rs5735 ( $>C$ ) showed lower 24-h urinary sodium excretion than those with either the TT or TC genotype. Individuals homozygous for the C allele of rs6497657 (T>C) showed lower 24-h urinary sodium excretion than those with either the TT or TC genotype. In addition, as using the general linear model with an additive model after adjusting for age, sex, BMI, and smoking status, 24-h urinary sodium excretion showed significant decreasing trends in the SCNN1G gene (rs4073291, rs12934362, rs7404408, rs4494543, rs5735, rs6497657, rs4260062, rs5740, rs4470152, rs4309398, and rs4341748). 
Table 3. Estimated 24-h urinary sodium excretion obtained with the Tanaka formula according to genotypes ${ }^{1}$.

\begin{tabular}{|c|c|c|c|c|c|c|}
\hline \multirow{2}{*}{ Gene } & \multirow{2}{*}{ SNP } & \multicolumn{3}{|c|}{ Genotype } & \multirow{2}{*}{$p$-Difference ${ }^{2}$} & \multirow{2}{*}{$p$-Trend } \\
\hline & & MM & $\mathrm{Mm}$ & $\mathrm{mm}$ & & \\
\hline \multirow{4}{*}{ SCNN1A } & rs13306613 & $164.31 \pm 0.66$ & $163.10 \pm 2.10$ & $157.66 \pm 11.25$ & 0.7259 & 0.8506 \\
\hline & rs7956915 & $163.00 \pm 1.00$ & $164.43 \pm 0.92$ & $166.73 \pm 1.66$ & 0.1462 & 0.0926 \\
\hline & rs4149621 & $164.19 \pm 0.69$ & $162.84 \pm 2.34$ & $155.19 \pm 17.84$ & 0.7567 & 0.3700 \\
\hline & rs11064153 & $164.60 \pm 0.97$ & $163.49 \pm 0.92$ & $165.39 \pm 1.78$ & 0.5431 & 0.9487 \\
\hline \multirow{8}{*}{ SCNN1B } & rs7205273 & $164.15 \pm 0.87$ & $164.14 \pm 0.99$ & $164.58 \pm 2.15$ & 0.9816 & 0.0521 \\
\hline & rs7190829 & $164.59 \pm 0.78$ & $163.54 \pm 1.12$ & $162.76 \pm 2.94$ & 0.6544 & 0.5149 \\
\hline & rs8055868 & $164.71 \pm 0.78$ & $163.05 \pm 1.13$ & $164.13 \pm 2.88$ & 0.4768 & 0.3728 \\
\hline & rs8044970 & $163.36 \pm 1.11$ & $164.59 \pm 0.90$ & $164.78 \pm 1.41$ & 0.6282 & 0.9313 \\
\hline & rs152733 & $164.27 \pm 1.09$ & $164.04 \pm 0.89$ & $164.41 \pm 1.46$ & 0.9722 & 0.4009 \\
\hline & rs239350 & $164.16 \pm 0.70$ & $164.51 \pm 1.94$ & $151.62 \pm 9.81$ & 0.4347 & 0.3109 \\
\hline & rs889299 & $164.12 \pm 0.88$ & $164.13 \pm 0.98$ & $165.02 \pm 2.12$ & 0.9214 & 0.3726 \\
\hline & rs11074555 & $164.20 \pm 0.83$ & $164.55 \pm 1.04$ & $161.94 \pm 2.45$ & 0.6170 & 0.0774 \\
\hline \multirow{22}{*}{ SCNN1G } & rs4073291 & $164.43 \pm 0.73^{\text {a } 3}$ & $164.37 \pm 1.28^{a}$ & $152.25 \pm 4.41^{b}$ & $0.0241^{4}$ & 0.0279 \\
\hline & rs12934362 & $164.45 \pm 0.73^{\mathrm{a}}$ & $164.79 \pm 1.31^{\mathrm{a}}$ & $153.02 \pm 4.57^{b}$ & 0.0431 & 0.0331 \\
\hline & rs7404408 & $164.44 \pm 0.73^{a}$ & $164.53 \pm 1.29^{a}$ & $152.26 \pm 4.42^{b}$ & 0.0237 & 0.0362 \\
\hline & rs4494543 & $164.44 \pm 0.73^{\mathrm{a}}$ & $164.53 \pm 1.29^{a}$ & $152.26 \pm 4.42^{b}$ & 0.0237 & 0.0362 \\
\hline & rs5735 & $164.38 \pm 0.73^{a}$ & $164.78 \pm 1.34^{\mathrm{a}}$ & $151.93 \pm 4.69^{b}$ & 0.0290 & 0.0209 \\
\hline & rs6497657 & $164.36 \pm 0.73^{a}$ & $164.62 \pm 1.36^{\mathrm{a}}$ & $151.06 \pm 4.74^{b}$ & 0.0198 & 0.0288 \\
\hline & rs4299163 & $163.82 \pm 0.69$ & $163.91 \pm 2.23$ & $152.47 \pm 13.46$ & 0.7003 & 0.1345 \\
\hline & rs11643777 & $164.01 \pm 0.67$ & $166.33 \pm 2.22$ & $149.31 \pm 17.79$ & 0.4261 & 0.5863 \\
\hline & rs4260062 & $163.82 \pm 0.69$ & $163.44 \pm 2.01$ & $155.58 \pm 12.57$ & 0.7952 & 0.0363 \\
\hline & rs5740 & $163.83 \pm 0.69$ & $163.47 \pm 1.98$ & $159.05 \pm 11.85$ & 0.9096 & 0.0432 \\
\hline & rs4499238 & $163.93 \pm 0.69$ & $165.29 \pm 1.63$ & $160.32 \pm 7.58$ & 0.6561 & 0.2301 \\
\hline & rs4470152 & $163.83 \pm 0.69$ & $163.39 \pm 1.85$ & $160.11 \pm 10.72$ & 0.9202 & 0.0497 \\
\hline & rs4309398 & $163.83 \pm 0.69$ & $163.44 \pm 1.86$ & $158.62 \pm 11.25$ & 0.8827 & 0.0351 \\
\hline & rs4341748 & $163.83 \pm 0.69$ & $163.34 \pm 1.86$ & $158.61 \pm 11.25$ & 0.8735 & 0.0319 \\
\hline & rs11648257 & $163.97 \pm 0.67$ & $166.05 \pm 1.92$ & $151.15 \pm 12.57$ & 0.3458 & 0.7657 \\
\hline & rs9941210 & $163.96 \pm 0.67$ & $166.04 \pm 1.92$ & $151.15 \pm 12.56$ & 0.3467 & 0.7642 \\
\hline & rs4499239 & $163.97 \pm 0.67$ & $165.98 \pm 1.92$ & $151.15 \pm 12.56$ & 0.3567 & 0.7705 \\
\hline & rs13306653 & $163.97 \pm 0.67$ & $166.56 \pm 1.89$ & $151.16 \pm 12.58$ & 0.2524 & 0.9566 \\
\hline & rs11643517 & $163.96 \pm 0.67$ & $166.31 \pm 1.88$ & $151.16 \pm 12.58$ & 0.2919 & 0.9887 \\
\hline & rs5723 & $163.95 \pm 0.67$ & $166.43 \pm 1.87$ & $151.17 \pm 12.57$ & 0.2681 & 0.9409 \\
\hline & rs3026 & $163.95 \pm 0.67$ & $166.43 \pm 1.87$ & $151.17 \pm 12.57$ & 0.2668 & 0.9361 \\
\hline & rs9930846 & $163.95 \pm 0.67$ & $166.43 \pm 1.87$ & $151.17 \pm 12.57$ & 0.2668 & 0.9361 \\
\hline
\end{tabular}

\footnotetext{
${ }^{1}$ All values are presented as the mean $\pm \mathrm{SD}$ (mEq/day); ${ }^{2}$ Values were derived using a general linear model analysis adjusted for age, sex, BMI, and smoking status; ${ }^{3}$ Values with different superscript letters within a row are significantly different $(p<0.05)$ among groups by Tukey's multiple comparison test; ${ }^{4}$ The significant $p$ value was denoted in bold; MM: major allele homozygous; Mm: heterozygous; mm: minor allele homozygous.
}

The associations of genetic variation in the $\mathrm{ENaC}$ gene with systolic blood pressure and diastolic blood pressure are shown in Tables 4 and 5, respectively. No differences were found in systolic blood pressure and diastolic blood pressure among the genotype groups.

Table 4. Associations between systolic blood pressure and genotypes ${ }^{1}$.

\begin{tabular}{|c|c|c|c|c|c|c|}
\hline \multirow{2}{*}{ Gene } & \multirow{2}{*}{ SNP } & \multicolumn{3}{|c|}{ Genotype } & \multirow{2}{*}{$p$-Difference ${ }^{2}$} & \multirow{2}{*}{$p$-Trend } \\
\hline & & MM & Mm & $\mathrm{mm}$ & & \\
\hline \multirow{4}{*}{ SCNN1A } & rs13306613 & $114.99 \pm 0.28$ & $114.36 \pm 0.89$ & $114.38 \pm 4.80$ & 0.7928 & 0.5030 \\
\hline & rs7956915 & $115.11 \pm 0.43$ & $114.57 \pm 0.39$ & $115.66 \pm 0.71$ & 0.3507 & 0.8536 \\
\hline & rs4149621 & $114.86 \pm 0.29$ & $113.99 \pm 0.99$ & $108.12 \pm 7.57$ & 0.4773 & 0.3007 \\
\hline & rs11064153 & $114.71 \pm 0.41$ & $115.09 \pm 0.39$ & $115.09 \pm 0.76$ & 0.7767 & 0.5310 \\
\hline
\end{tabular}


Table 4. Cont.

\begin{tabular}{|c|c|c|c|c|c|c|}
\hline \multirow{2}{*}{ Gene } & \multirow{2}{*}{ SNP } & \multicolumn{3}{|c|}{ Genotype } & \multirow{2}{*}{$p$-Difference ${ }^{2}$} & \multirow{2}{*}{$p$-Trend } \\
\hline & & MM & Mm & $\mathbf{m m}$ & & \\
\hline \multirow{8}{*}{ SCNN1B } & rs7205273 & $115.16 \pm 0.37$ & $114.84 \pm 0.42$ & $113.96 \pm 0.92$ & 0.4596 & 0.2428 \\
\hline & rs7190829 & $115.16 \pm 0.33$ & $114.43 \pm 0.48$ & $115.12 \pm 1.26$ & 0.4372 & 0.3529 \\
\hline & rs8055868 & $115.14 \pm 0.33$ & $114.29 \pm 0.48$ & $116.31 \pm 1.23$ & 0.1789 & 0.6593 \\
\hline & rs 8044970 & $115.25 \pm 0.47$ & $114.85 \pm 0.38$ & $114.59 \pm 0.60$ & 0.6646 & 0.3710 \\
\hline & rs152733 & $114.69 \pm 0.47$ & $115.02 \pm 0.38$ & $115.11 \pm 0.62$ & 0.8160 & 0.5502 \\
\hline & rs239350 & $114.86 \pm 0.30$ & $115.45 \pm 0.83$ & $112.29 \pm 4.22$ & 0.6594 & 0.6956 \\
\hline & rs889299 & $115.05 \pm 0.38$ & $114.83 \pm 0.42$ & $114.63 \pm 0.91$ & 0.8739 & 0.6036 \\
\hline & rs11074555 & $114.81 \pm 0.35$ & $115.39 \pm 0.44$ & $113.48 \pm 1.05$ & 0.2107 & 0.8865 \\
\hline \multirow{22}{*}{ SCNN1G } & rs4073291 & $114.75 \pm 0.31$ & $115.43 \pm 0.55$ & $115.61 \pm 1.88$ & 0.5250 & 0.2658 \\
\hline & rs12934362 & $114.80 \pm 0.31$ & $115.46 \pm 0.56$ & $116.20 \pm 1.94$ & 0.4706 & 0.2196 \\
\hline & rs7404408 & $114.77 \pm 0.31$ & $115.45 \pm 0.55$ & $115.62 \pm 1.88$ & 0.5213 & 0.2637 \\
\hline & rs4494543 & $114.77 \pm 0.31$ & $115.45 \pm 0.55$ & $115.62 \pm 1.88$ & 0.5213 & 0.2637 \\
\hline & rs5735 & $114.78 \pm 0.31$ & $115.39 \pm 0.57$ & $116.48 \pm 1.99$ & 0.4694 & 0.2250 \\
\hline & rs6497657 & $114.78 \pm 0.31$ & $115.22 \pm 0.58$ & $116.12 \pm 2.01$ & 0.6577 & 0.3715 \\
\hline & rs4299163 & $114.97 \pm 0.29$ & $114.78 \pm 0.95$ & $112.20 \pm 5.73$ & 0.8755 & 0.7415 \\
\hline & rs11643777 & $114.94 \pm 0.29$ & $115.30 \pm 0.95$ & $110.52 \pm 7.60$ & 0.7898 & 0.8350 \\
\hline & rs4260062 & $114.95 \pm 0.29$ & $114.17 \pm 0.86$ & $112.04 \pm 5.35$ & 0.6009 & 0.3270 \\
\hline & rs5740 & $114.95 \pm 0.29$ & $114.35 \pm 0.85$ & $114.15 \pm 5.05$ & 0.7879 & 0.4925 \\
\hline & rs4499238 & $115.01 \pm 0.30$ & $114.81 \pm 0.70$ & $111.91 \pm 3.25$ & 0.6187 & 0.5358 \\
\hline & rs4470152 & $114.93 \pm 0.29$ & $114.20 \pm 0.79$ & $114.37 \pm 4.56$ & 0.6794 & 0.3903 \\
\hline & rs4309398 & $114.93 \pm 0.29$ & $114.25 \pm 0.79$ & $113.17 \pm 4.79$ & 0.6764 & 0.3784 \\
\hline & rs4341748 & $114.93 \pm 0.29$ & $114.30 \pm 0.79$ & $113.18 \pm 4.79$ & 0.7057 & 0.4068 \\
\hline & rs11648257 & $114.92 \pm 0.29$ & $115.10 \pm 0.82$ & $109.26 \pm 5.37$ & 0.5605 & 0.9094 \\
\hline & rs9941210 & $114.92 \pm 0.29$ & $115.09 \pm 0.82$ & $109.26 \pm 5.37$ & 0.5622 & 0.8986 \\
\hline & rs4499239 & $114.92 \pm 0.29$ & $115.05 \pm 0.82$ & $109.26 \pm 5.37$ & 0.5668 & 0.8635 \\
\hline & rs13306653 & $114.92 \pm 0.29$ & $115.08 \pm 0.81$ & $109.25 \pm 5.37$ & 0.5615 & 0.8993 \\
\hline & rs11643517 & $114.90 \pm 0.29$ & $115.03 \pm 0.80$ & $109.23 \pm 5.36$ & 0.5638 & 0.8768 \\
\hline & rs5723 & $114.90 \pm 0.29$ & $115.12 \pm 0.80$ & $109.23 \pm 5.36$ & 0.5502 & 0.9617 \\
\hline & rs3026 & $114.91 \pm 0.29$ & $115.13 \pm 0.80$ & $109.24 \pm 5.36$ & 0.5514 & 0.9547 \\
\hline & rs9930846 & $114.91 \pm 0.29$ & $115.13 \pm 0.80$ & $109.24 \pm 5.36$ & 0.5514 & 0.9547 \\
\hline
\end{tabular}

${ }^{1}$ All values are presented as the mean $\pm \mathrm{SD}(\mathrm{mmHg}) ;{ }^{2}$ Values were derived using a general linear model analysis adjusted for age, sex, BMI, and smoking status; MM: major allele homozygous; Mm: heterozygous; mm: minor allele homozygous.

Table 5. Associations between diastolic blood pressure and genotypes ${ }^{1}$.

\begin{tabular}{|c|c|c|c|c|c|c|}
\hline \multirow{2}{*}{ Gene } & \multirow{2}{*}{ SNP } & \multicolumn{3}{|c|}{ Genotype } & \multirow{2}{*}{$p$-Difference ${ }^{2}$} & \multirow{2}{*}{$p$-Trend } \\
\hline & & MM & $\mathrm{Mm}$ & $\mathrm{mm}$ & & \\
\hline \multirow{4}{*}{ SCNN1A } & rs13306613 & $73.76 \pm 0.19$ & $72.43 \pm 0.61$ & $75.20 \pm 3.26$ & 0.1004 & 0.0728 \\
\hline & rs7956915 & $73.57 \pm 0.29$ & $73.54 \pm 0.27$ & $74.22 \pm 0.48$ & 0.4435 & 0.3752 \\
\hline & rs4149621 & $73.63 \pm 0.20$ & $73.05 \pm 0.67$ & $69.28 \pm 5.15$ & 0.4974 & 0.3078 \\
\hline & rs11064153 & $73.49 \pm 0.28$ & $73.66 \pm 0.27$ & $74.13 \pm 0.51$ & 0.5535 & 0.3084 \\
\hline \multirow{8}{*}{ SCNN1B } & rs7205273 & $73.80 \pm 0.25$ & $73.56 \pm 0.29$ & $73.12 \pm 0.62$ & 0.5585 & 0.2917 \\
\hline & rs7190829 & $73.75 \pm 0.23$ & $73.39 \pm 0.32$ & $73.99 \pm 0.85$ & 0.6028 & 0.6225 \\
\hline & rs8055868 & $73.70 \pm 0.23$ & $73.40 \pm 0.33$ & $74.75 \pm 0.83$ & 0.3014 & 0.8278 \\
\hline & rs8044970 & $73.63 \pm 0.32$ & $73.70 \pm 0.26$ & $73.49 \pm 0.41$ & 0.9067 & 0.8237 \\
\hline & rs152733 & $73.40 \pm 0.32$ & $73.87 \pm 0.26$ & $73.49 \pm 0.42$ & 0.4675 & 0.6841 \\
\hline & rs239350 & $73.61 \pm 0.20$ & $73.88 \pm 0.56$ & $72.55 \pm 2.85$ & 0.8360 & 0.7733 \\
\hline & rs889299 & $73.75 \pm 0.25$ & $73.53 \pm 0.28$ & $73.54 \pm 0.62$ & 0.8214 & 0.5754 \\
\hline & rs11074555 & $73.74 \pm 0.24$ & $73.67 \pm 0.30$ & $72.72 \pm 0.71$ & 0.3968 & 0.3177 \\
\hline
\end{tabular}


Table 5. Cont

\begin{tabular}{|c|c|c|c|c|c|c|}
\hline \multirow{2}{*}{ Gene } & \multirow{2}{*}{ SNP } & \multicolumn{3}{|c|}{ Genotype } & \multirow{2}{*}{$p$-Difference ${ }^{2}$} & \multirow{2}{*}{$p$-Trenc } \\
\hline & & MM & Mm & $\mathrm{mm}$ & & \\
\hline \multirow{22}{*}{ SCNN1G } & rs4073291 & $73.63 \pm 0.21$ & $73.61 \pm 0.37$ & $74.66 \pm 1.28$ & 0.7254 & 0.7256 \\
\hline & rs12934362 & $73.65 \pm 0.21$ & $73.78 \pm 0.38$ & $75.16 \pm 1.31$ & 0.5145 & 0.4168 \\
\hline & rs7404408 & $73.64 \pm 0.21$ & $73.64 \pm 0.37$ & $74.67 \pm 1.28$ & 0.7285 & 0.6950 \\
\hline & rs4494543 & $73.64 \pm 0.21$ & $73.64 \pm 0.37$ & $74.67 \pm 1.28$ & 0.7285 & 0.6950 \\
\hline & rs5735 & $73.65 \pm 0.21$ & $73.76 \pm 0.39$ & $74.99 \pm 1.35$ & 0.6094 & 0.4860 \\
\hline & rs6497657 & $73.64 \pm 0.21$ & $73.77 \pm 0.39$ & $74.74 \pm 1.36$ & 0.7020 & 0.5164 \\
\hline & rs4299163 & $73.72 \pm 0.20$ & $73.47 \pm 0.65$ & $72.98 \pm 3.92$ & 0.9172 & 0.6805 \\
\hline & rs11643777 & $73.72 \pm 0.19$ & $73.62 \pm 0.64$ & $70.41 \pm 5.14$ & 0.8056 & 0.7673 \\
\hline & rs4260062 & $73.71 \pm 0.20$ & $73.15 \pm 0.59$ & $72.60 \pm 3.66$ & 0.6350 & 0.3405 \\
\hline & rs5740 & $73.71 \pm 0.20$ & $73.25 \pm 0.58$ & $72.55 \pm 3.46$ & 0.7146 & 0.4139 \\
\hline & rs4499238 & $73.73 \pm 0.20$ & $73.27 \pm 0.47$ & $71.72 \pm 2.20$ & 0.4578 & 0.2441 \\
\hline & rs4470152 & $73.70 \pm 0.20$ & $73.18 \pm 0.54$ & $72.76 \pm 3.12$ & 0.6310 & 0.3374 \\
\hline & rs4309398 & $73.71 \pm 0.20$ & $73.17 \pm 0.54$ & $71.94 \pm 3.27$ & 0.5620 & 0.2915 \\
\hline & rs4341748 & $73.71 \pm 0.20$ & $73.18 \pm 0.54$ & $71.94 \pm 3.27$ & 0.5771 & 0.3041 \\
\hline & rs11648257 & $73.71 \pm 0.19$ & $73.20 \pm 0.56$ & $69.92 \pm 3.65$ & 0.4045 & 0.2567 \\
\hline & rs9941210 & $73.71 \pm 0.19$ & $73.20 \pm 0.56$ & $69.92 \pm 3.65$ & 0.4060 & 0.2586 \\
\hline & rs4499239 & $73.72 \pm 0.19$ & $73.21 \pm 0.56$ & $69.92 \pm 3.65$ & 0.4099 & 0.2633 \\
\hline & rs13306653 & $73.71 \pm 0.19$ & $73.20 \pm 0.55$ & $69.92 \pm 3.65$ & 0.4018 & 0.2555 \\
\hline & rs11643517 & $73.71 \pm 0.19$ & $73.19 \pm 0.54$ & $69.90 \pm 3.64$ & 0.3956 & 0.2494 \\
\hline & rs5723 & $73.7 \pm 0.19$ & $73.19 \pm 0.54$ & $69.90 \pm 3.64$ & 0.3966 & 0.2503 \\
\hline & rs3026 & $73.71 \pm 0.19$ & $73.19 \pm 0.54$ & $69.91 \pm 3.64$ & 0.3941 & 0.2477 \\
\hline & rs9930846 & $73.71 \pm 0.19$ & $73.19 \pm 0.54$ & $69.91 \pm 3.64$ & 0.3941 & 0.2477 \\
\hline
\end{tabular}

${ }^{1}$ All values are presented as the mean $\pm \mathrm{SD}(\mathrm{mmHg}) ;{ }^{2}$ Values were derived using a general linear model analysis adjusted for age, sex, BMI, and smoking status; MM: major allele homozygous; Mm: heterozygous; mm: minor allele homozygous.

\section{Discussion}

The present study was performed to determine the association of genetic variation in ENaC (SCNN1A, SCNN1B, and SCNN1G) with urinary sodium excretion and blood pressure in Korean adults. Polymorphisms of six SNPs in the genes that code for the ENaC $\gamma$ subunit (SCNN1G) may modify urinary sodium excretion. However, no difference was found in blood pressure among the gene variants of ENaC.

Homozygotes for the minor alleles of all SNPs in the SCNN1G gene showed lower 24-h urinary sodium excretion than carriers of major alleles. Among them, six SNPs (rs4073291, rs12934362, rs7404408, rs4494543, rs5735, and rs6497657) were significantly different in 24-h urinary sodium excretion and 11 SNPs (rs4073291, rs12934362, rs7404408, rs4494543, rs5735, rs6497657, rs4260062, rs5740, rs4470152, rs4309398, and rs4341748) showed significant decreasing trends in an additive model. However, there was no difference in the SCNN1A and SCNN1B genes.

$\mathrm{ENaC}$ is located in the distal nephron. Aldosterone and vasopressin regulate ENaC activity. A low-sodium diet stimulates the renin-angiotensin-aldosterone system (RAAS), and aldosterone increases sodium reabsorption by activating $\mathrm{ENaC}$ in the distal nephron. Vasopressin is secreted in response to increases in plasma osmolality, which means a water deficit. Vasopressin stimulates ENaC activity and increases water conservation by increasing sodium reabsorption [4]. In addition, proteolytic ENaC activation by serine proteases may contribute to sodium reabsorption [17-19]. Proteases cleave specific sites in the extracellular domains of the $\alpha$ - and $\gamma$-subunits [17-19].

Low 24-h urinary sodium excretion can be associated with high sodium reabsorption in the kidney or low sodium intake. Thus, the persons who are homozygous for the minor alleles of six SNPs (rs4073291, rs12934362, rs7404408, rs4494543, rs5735, and rs6497657) of the SCNN1G gene may reabsorb more sodium in the kidney or consume less sodium than the persons having carriers of major alleles. Excessive sodium reabsorption by the kidney has been known to increase the risk of hypertension. In a South African study, there were no differences in urinary sodium excretion between the wild type 
and carriers of the R563Q variant of the ENaC [20]. However, the G442V polymorphism in SCNN1B showed greater $\mathrm{Na}$ retention in normotensive young people (both black and white) [21]. In addition, Vormfelde SV et al. reported that carriers of the variant G-allele of rs5723 in SCNN1G tended to excrete less sodium in healthy white adults [22]. Therefore, variants of ENaC, particularly SCNN1B and SCNN1G, may be associated with reabsorption of more sodium, but there may be racial differences.

Twenty-four-hour urinary sodium excretion can reflect dietary sodium intake; thus, the people who are homozygous for the minor alleles of SNPs in the SCNN1G may consume less sodium than those who are carriers of major alleles. These differences seem to be related to taste perception. Salt taste perception has been the focus of studies on salt taste receptors such as the ENaC and transient receptor potential cation channel subfamily $\mathrm{V}$ member 1 (TRPV1) [23]. ENaC is located within taste cell membranes and is the key mediator of salt taste. In the study by Dias et al. [24], two SNPs (rs239345 (A>T) and rs3785368 (C>T)) in intronic regions within SCNN1B modified suprathreshold taste sensitivity. In the TRPV1 gene, one SNP (rs8065080 (C>T)) polymorphism modified salt taste perception. Carriers of the $\mathrm{T}$ allele perceived salt solutions stronger than those homozygous for the $\mathrm{C}$ allele. Even though the types of taste receptors were not the same as in the present study, genetic variation in salt taste receptors seems to modify taste perception, which results in the differences in salt intake. However, according to a twin study, salt taste perception appears to be determined more by environmental influences [25]. Further clinical trials on salt taste perception are needed.

In the present study, the SCNN1G gene was associated with 24-h urinary sodium excretion but was not related to blood pressure. The SCNN1A and SCNN1B genes were not associated with blood pressure either. The present study was performed to focus on urinary sodium excretion reflecting salt intake. Hypertensive patients were excluded from the study since they tried to modify their sodium intake. Previous studies reported conflicting results about the associations of ENaC with blood pressure [11,26-29]. The Genetic Epidemiology Network of Salt Sensitivity (GenSalt) study [26] conducted in the Han Chinese population reported that rs13306613 in the SCNN1A gene was associated with diastolic blood pressure (DBP) and that rs12447134 in the SCNN1B gene was associated with systolic blood pressure (SBP) under a codominant model. In addition, 5 SNPs in SCNN1G and 4 SNPs in SCNN1B were associated with SBP, DBP, or mean arterial pressure (MAP) under the additive model. In addition, in the other GenSalt study, SCNN1A SNP rs11064153 and SCNN1G SNP rs4401050 were related to longitudinal changes in SBP [11]. However, the T594M variant of the ENaC gene was not associated with hypertension among individuals of African ancestry [27] and Indo-Aryan ancestry [28]. The $\gamma 649$ ENaC polymorphism was not related to hypertension or salt sensitivity either [29].

There are some limitations to consider when interpreting the results of this study. First, 24-h urinary sodium excretion was estimated using the Tanaka formula with spot urine samples. The 24-h urine collection is hardly realistic in large-sample epidemiologic studies. Second, sodium reabsorption in the kidney is affected by hormones, such as aldosterone and vasopressin, but they were not measured in the present study. Third, sodium intake and salt perception were not measured; thus, it is not known whether the difference in urinary sodium excretion according to ENaC gene variants is due to sodium intake (salt taste perception) or sodium reabsorption in the kidney. Fourth, persons with hypertension were excluded in the present study because hypertension patients tended to try a low sodium diet and we were more interested in sodium intake according to gene variants.

Despite these limitations, this study confirmed that there was a difference in 24-urinary sodium excretion according to variants of the SCNN1G gene in large samples, and this difference did not appear for blood pressure. Further studies are needed to determine whether the difference in 24-h urinary sodium excretion according to the $\mathrm{ENaC}$ gene variant is due to salt taste perception differences or sodium reabsorption differences in the kidney. In addition, since ENaC activity is also related to urinary potassium excretion, urinary $\mathrm{Na} / \mathrm{K}$ ratio should be compared by genetic variation in the $\mathrm{ENaC}$ gene. 
Author Contributions: Y.J.Y. and C.K.K. conceived and designed the experiments; Y.J.Y. and J.K. analyzed the data; C.K.K., Y.J.Y. and J.K. contributed to the oversight of the study and gave significant comments; Y.J.Y. and J.K. wrote the paper.

Funding: This research was funded by the Korea Food Research Institute (E0150308-04).

Acknowledgments: This study was provided with bioresources from the National Biobank of Korea, the Centers for Disease Control and Prevention, Republic of Korea (4845-301, 4851-302 and -307). This research was funded by the Korea Food Research Institute (E0150308-04).

Conflicts of Interest: The authors declare no conflict of interest.

\section{References}

1. DeSimone, J.A.; Lyall, V. Taste receptors in the gastrointestinal tract III. Salty and sour taste: Sensing of sodium and protons by the tongue. Am. J. Physiol. Gastrointest. Liver Physiol. 2006, 291, G1005-G1010. [CrossRef] [PubMed]

2. Chandrashekar, J.; Kuhn, C.; Oka, Y.; Yarmolinsky, D.A.; Hummler, E.; Ryba, N.J.; Zuker, C.S. The cells and peripheral representation of sodium taste in mice. Nature 2010, 464, 297-301. [CrossRef] [PubMed]

3. Thomas, C.P.; Itani, O.A. New insights into epithelial sodium channel function in the kidney: Site of action, regulation by ubiquitin ligases, serum- and glucocorticoid-inducible kinase and proteolysis. Curr. Opin. Nephrol. Hypertens. 2004, 13, 541-548. [CrossRef] [PubMed]

4. Bankir, L.; Bichet, D.G.; Bouby, N. Vasopressin V2 receptors, ENaC, and sodium reabsorption: A risk factor for hypertension? Am. J. Physiol. Ren. Physiol. 2010, 299, F917-F928. [CrossRef] [PubMed]

5. Huppert, L.A.; Matthay, M.A. Alveolar fluid clearance in pathologically relevant conditions: In vitro and in vivo models of acute respiratory distress syndrome. Front. Immunol. 2017, 8, 371. [CrossRef] [PubMed]

6. Li, W.; Long, C.; Renjun, L.; Zhangxue, H.; Yin, H.; Wanwei, L.; Juan, M.; Yuan, S. Association of SCNN1A Single Nucleotide Polymorphisms with neonatal respiratory distress syndrome. Sci. Rep. 2015, 5, 17317. [CrossRef] [PubMed]

7. Kellenberger, S.; Schild, L. Epithelial sodium channel/degenerin family of ion channels: A variety of functions for a shared structure. Physiol. Rev. 2002, 82, 735-767. [CrossRef] [PubMed]

8. Shimkets, R.A.; Warnock, D.G.; Bositis, C.M.; Nelson-Williams, C.; Hansson, J.H.; Schambelan, M.; Gill, J.R., Jr.; Ulick, S.; Milora, R.V.; Findling, J.W.; et al. Liddle's syndrome: Heritable human hypertension caused by mutations in the beta subunit of the epithelial sodium channel. Cell 1994, 79, 407-414. [CrossRef]

9. Garcia-Bailo, B.; Toguri, C.; Eny, K.M.; El-Sohemy, A. Genetic variation in taste and its influence on food selection. Omics J. Integr. Biol. 2009, 13, 69-80. [CrossRef] [PubMed]

10. Folkesson, H.G.; Matthay, M.A. Alveolar epithelial ion and fluid transport: Recent progress. Am. J. Respir. Cell Mol. Biol. 2006, 35, 10-19. [CrossRef] [PubMed]

11. Yang, X.; He, J.; Gu, D.; Hixson, J.E.; Huang, J.; Rao, D.C.; Shimmin, L.C.; Chen, J.; Rice, T.K.; Li, J.; et al. Associations of epithelial sodium channel genes with blood pressure changes and hypertension incidence: The GenSalt study. Am. J. Hypertens. 2014, 27, 1370-1376. [CrossRef] [PubMed]

12. Kim, Y.; Han, B.G. Cohort profile: The Korean Genome and Epidemiology Study (KoGES) Consortium. Int. J. Epidemiol. 2017, 46, e20. [CrossRef] [PubMed]

13. Cho, Y.S.; Go, M.J.; Kim, Y.J.; Heo, J.Y.; Oh, J.H.; Ban, H.J.; Yoon, D.; Lee, M.H.; Kim, D.J.; Park, M.; et al. A large-scale genome-wide association study of Asian populations uncovers genetic factors influencing eight quantitative traits. Nat. Genet. 2009, 41, 527-534. [CrossRef] [PubMed]

14. Tanaka, T.; Okamura, T.; Miura, K.; Kadowaki, T.; Ueshima, H.; Nakagawa, H.; Hashimoto, T. A simple method to estimate populational 24-h urinary sodium and potassium excretion using a casual urine specimen. J. Hum. Hypertens. 2002, 16, 97-103. [CrossRef] [PubMed]

15. Marchini, J.; Howie, B.; Myers, S.; McVean, G.; Donnelly, P. A new multipoint method for genome-wide association studies by imputation of genotypes. Nat. Genet. 2007, 39, 906-913. [CrossRef] [PubMed]

16. Wellcome Trust Case Control Consortium. Genome-wide association study of 14,000 cases of seven common diseases and 3000 shared controls. Nature 2007, 447, 661-678.

17. Bruns, J.B.; Carattino, M.D.; Sheng, S.; Maarouf, A.B.; Weisz, O.A.; Pilewski, J.M.; Hughey, R.P.; Kleyman, T.R. Epithelial $\mathrm{Na}+$ channels are fully activated by furin- and prostasin-dependent release of an inhibitory peptide from the gamma-subunit. J. Biol. Chem. 2007, 282, 6153-6160. [CrossRef] [PubMed] 
18. Carattino, M.D.; Sheng, S.; Bruns, J.B.; Pilewski, J.M.; Hughey, R.P.; Kleyman, T.R. The epithelial Na+ channel is inhibited by a peptide derived from proteolytic processing of its alpha subunit. J. Biol. Chem. 2006, 281, 18901-18907. [CrossRef] [PubMed]

19. Kleyman, T.R.; Carattino, M.D.; Hughey, R.P. ENaC at the cutting edge: Regulation of epithelial sodium channels by proteases. J. Biol. Chem. 2009, 284, 20447-20451. [CrossRef] [PubMed]

20. Jones, E.S.; Owen, E.P.; Rayner, B.L. The association of the R563Q genotype of the ENaC with phenotypic variation in Southern Africa. Am. J. Hypertens. 2012, 25, 1286-1291. [CrossRef] [PubMed]

21. Ambrosius, W.T.; Bloem, L.J.; Zhou, L.; Rebhun, J.F.; Snyder, P.M.; Wagner, M.A.; Guo, C.; Pratt, J.H. Genetic variants in the epithelial sodium channel in relation to aldosterone and potassium excretion and risk for hypertension. Hypertension 1999, 34, 631-637. [CrossRef] [PubMed]

22. Vormfelde, S.V.; Sehrt, D.; Toliat, M.R.; Schirmer, M.; Meineke, I.; Tzvetkov, M.; Nurnberg, P.; Brockmoller, J. Genetic variation in the renal sodium transporters NKCC2, NCC, and ENaC in relation to the effects of loop diuretic drugs. Clin. Pharmacol. Ther. 2007, 82, 300-309. [CrossRef] [PubMed]

23. Chamoun, E.; Mutch, D.M.; Allen-Vercoe, E.; Buchholz, A.C.; Duncan, A.M.; Spriet, L.L.; Haines, J.; Ma, D.W.L. A review of the associations between single nucleotide polymorphisms in taste receptors, eating behaviors, and health. Crit. Rev. Food Sci. Nutr. 2018, 58, 194-207. [CrossRef] [PubMed]

24. Dias, A.G.; Rousseau, D.; Duizer, L.; Cockburn, M.; Chiu, W.; Nielsen, D.; El-Sohemy, A. Genetic variation in putative salt taste receptors and salt taste perception in humans. Chem. Senses 2013, 38, 137-145. [CrossRef] [PubMed]

25. Wise, P.M.; Hansen, J.L.; Reed, D.R.; Breslin, P.A. Twin study of the heritability of recognition thresholds for sour and salty taste. Chem. Senses 2007, 32, 749-754. [CrossRef] [PubMed]

26. Liu, F.; Yang, X.; Mo, X.; Huang, J.; Chen, J.; Kelly, T.N.; Hixson, J.E.; Rao, D.C.; Gu, C.C.; Shimmin, L.C.; et al. Associations of epithelial sodium channel genes with blood pressure: The GenSalt study. J. Hum. Hypertens. 2015, 29, 224-228. [CrossRef] [PubMed]

27. Nkeh, B.; Samani, N.J.; Badenhorst, D.; Libhaber, E.; Sareli, P.; Norton, G.R.; Woodiwiss, A.J. T594M variant of the epithelial sodium channel beta-subunit gene and hypertension in individuals of African ancestry in South Africa. Am. J. Hypertens. 2003, 16, 847-852. [CrossRef]

28. Gupta, M.D.; Girish, M.P.; Sikdar, S.; Ahuja, R.; Shah, D.; Kumar, R.; Rain, M.; Nejatizadeh, A.; Tyagi, S.; Pasha, Q. beta-T594M epithelial sodium channel gene polymorphism and essential hypertension in individuals of Indo-Aryan ancestry in Northern India. Indian Heart J. 2014, 66, 397-400. [CrossRef] [PubMed]

29. Poch, E.; Gonzalez, D.; de la Sierra, A.; Giner, V.; Bragulat, E.; Botey, A.; Coca, A.; Rivera, F. Genetic variation of the gamma subunit of the epithelial $\mathrm{Na}+$ channel and essential hypertension. Relationship with salt sensitivity. Am. J. Hypertens. 2000, 13, 648-653. [CrossRef] 